\title{
Erratum to: Characterising and correcting batch variation in an automated direct infusion mass spectrometry (DIMS) metabolomics workflow
}

\author{
J. A. Kirwan • D. I. Broadhurst • R. L. Davidson • \\ M. R. Viant
}

Received: 16 July 2014 / Accepted: 16 July 2014 / Published online: 30 July 2014

(C) Springer-Verlag Berlin Heidelberg 2014

Erratum to: Anal Bioanal Chem

DOI 10.1007/s00216-013-6856-7

The $\mathbf{X}^{\text {uncorr }}$ dataset described in the original manuscript was processed using a subtly different method to the other three datasets $\left(\mathrm{X}^{\mathrm{TIC}}, \mathrm{X}^{\mathrm{TIC}+\text { batch }}, \mathrm{X}^{\mathrm{TIC}+\text { batch+clean }}\right)$. The original processing of the $\mathbf{X}^{\mathbf{u n c o r r}}$ dataset included a step which removed outlying transients ( $<40 \%$ of the maximum total ion count) and used ten rather than nine blank samples to identify features that may not be of biological origin. Accordingly, the correctly processed $\mathbf{X}^{\text {uncorr }}$ dataset has now been used to recalculate the results throughout the manuscript which have changed minimally, and in several cases imperceptibly, from the original study. For completeness, however, we have reproduced all of the relevant tables and figures which the authors are happy to provide on request. Table 1 is reproduced below to demonstrate the extent of the difference between the original and the new results. Importantly, the conclusions drawn in the original study are still strongly supported by the revised findings.

The online version of the original article can be found at http://dx.doi.org/ 10.1007/s00216-013-6856-7.

J. A. Kirwan · M. R. Viant $(\bowtie)$

School of Biosciences, University of Birmingham, Edgbaston,

Birmingham B15 2TT, UK

e-mail: M.Viant@bham.ac.uk

D. I. Broadhurst

Department of Medicine, University of Alberta, Edmonton,

AB, Canada T6G 2EI

R. L. Davidson • M. R. Viant

NERC Biomolecular Analysis Facility-Metabolomics Node

(NBAF-B), University of Birmingham, Edgbaston,

Birmingham B15 2TT, UK 
Table 1 Analytical precision of the FT-ICR MS datasets presented as median $\mathrm{RSD}^{\mathrm{QC}}$ values (in percent), within each individual batch and across all eight batches, after each of the four stages of data processing.
Bolded values are those that are different between the original study and the corrected dataset.

Dataset

\begin{tabular}{|c|c|c|c|c|}
\hline $\mathrm{X}^{\mathrm{uncorr}}$ Corrected values & $\begin{array}{l}X^{\text {uncorr }} \\
\text { Erroneous values quoted } \\
\text { in original paper }\end{array}$ & $\mathrm{X}^{\mathrm{TIC}}$ & $\mathrm{X}^{\mathrm{TIC}+\text { batch }}$ & $\mathrm{X}^{\mathrm{TIC}+\text { batch+clean }}$ \\
\hline
\end{tabular}

\begin{tabular}{lrrrrr}
\hline Batch 1 & $\mathbf{9 . 2}$ & 8.5 & 8.7 & 7.3 & 6.9 \\
Batch 2 & $\mathbf{9 . 6}$ & 9.7 & 9.7 & 8.3 & 7.8 \\
Batch 3 & 7.8 & 7.8 & 7.4 & 6.3 & 6.0 \\
Batch 4 & 9.7 & 9.7 & 10.0 & 8.3 & 7.8 \\
Batch 5 & 8.5 & 8.5 & 8.5 & 4.9 & 8.3 \\
Batch 6 & 11.5 & 11.5 & 11.5 & 7.3 & 8.0 \\
Batch 7 & 8.9 & 8.9 & 9.0 & 7.2 & 7.1 \\
Batch 8 & 8.4 & 8.4 & 18.5 & & 7.0 \\
Batches 1 to 8 & $\mathbf{1 8 . 5}$ & 18.7 & & & 8.6 \\
\hline
\end{tabular}

